\title{
Keepered Media Recording: super-resolution for magnetic recording
}

\author{
Roger WOOD ${ }^{1}$, Tom COUGHLIN ${ }^{2}$, and James MONSON ${ }^{3}$ \\ 1) IBM Storage Systems Division, San Jose, California \\ 2) Ampex Corporation, Redwood City, California \\ 3) Harvey Mudd College, Claremont, California
}

\begin{abstract}
Keepered media are made up of two layers: a conventional hard longitudinal film plus a soft magnetic film or "keeper" layer. To recover the written data, a small DC bias current is applied to the inductive readback head. The nonlinear behavior of the soft film in the presence of the bias field provides the mechanism for super-resolution. This paper reviews the history and principles of keepered recording. The structure and fabrication of keepered media are described. Measurements are presented which show the improved resolution and amplitude and which quantify the noise, nonlinearity, and sidereading/sidewriting behavior. Several other "keepered" configurations including perpendicular options are described. Work is ongoing to optimize the performance of keepered media with MR heads. There is also evidence that the keeper stabilizes the stored data and will extend the superparamagnetic limit on areal recording density
\end{abstract}

\section{INTRODUCTION}

The hard disk-drive industry is currently maintaining a remarkable $60 \%$ per annum growth rate in information storage density. However, there is not a lot that is fundamentally new in magnetic recording technology. Today, just as fifty years ago, commercial products use a "ring" head writing on a "longitudinal" recording medium. The two most recent innovations in the Hard Disk Drive industry, magneto-resistive (MR) heads and the Partial-Response Maximum-Likelihood (PRML) $\mathrm{read} / \mathrm{write}$ channels were both invented nearly 30 years ago. Neither of these innovations alters the basic writing process or the storage mode. "Perpendicular" recording does represent a quite different recording technique. In two-layer perpendicular media a soft magnetic film is placed below the hard storage layer. This film, which is relatively thick, serves to reinforce and 'focus' the fields during write and also improves the head-efficiency during read. Perpendicular recording has been the focus of intense research, particularly in Japan. So far, however, perpendicular recording has not become commercially successful.

Keepered recording is a very novel approach which changes the storage and readback modes considerably. As in perpendicular recording, the recording medium includes a soft film. In keepered media, however, the soft film plays a very different role. In the typical keepered media configuration, an additional thin layer of magnetically soft film (a "keeper") is deposited on top of an otherwise conventional longitudinal medium, Figure
1. In order to recover the recorded information, a small DC bias current must be applied to the inductive readback head. Because of the bias, the thin soft film saturates locally in the proximity of the head gap. The nonlinear behavior of the soft film provides the mechanism for superresolution in the readback process. The reproduced pulses are found not only to be narrower but also to have increased amplitude.

The concept of keepered recording arose in 1984 [1,2] as one of a series of intriguing innovations arising from the work of Gooch at Ampex Corporation. Thin layers of soft saturable film were being proposed in a variety of applications ranging from electronic track-following to low wear-rate rotary scanners [3-6]. In the following years, however, work on keepered recording paused as activities on hard disk drives at Ampex ceased. Interest was revived at various times from 1990 to 1996 with a number of companies pursing the technique as an alternative to MR head technology [7,8]. In academia, the University of Minnesota has maintained an ongoing activity in both the theory and the implementation of keepered recording $[9,10]$. Recent work by Wilson et al. at Stanford University has focused on overcoming some of the nonlinear distortions inherent in the technique [11].

The work of Sawazaki bears some relationship to keepered recording. Sawazaki proposed that soft magnetic material be included into the tape medium but that AC-bias (rather than a DC-bias) be used in a parametric readback mode $[12,13]$. Also Mallary has proposed using a thick underlying soft film spaced away from the hard film as a means of reducing sidereading effects [14].

In an interesting analog of keepered recording, Murakami et al. [15] describe a magneto-optical system giving superresolution in which the anisotropy of a soft overlayer is switched by heat from an incident laser spot.

Keepered recording has not yet appeared in any commercial products. However, interest in the technology has recently been rekindled by the recognition that this storage mode is intrinsically very stable. At very high areal densities approaching the 


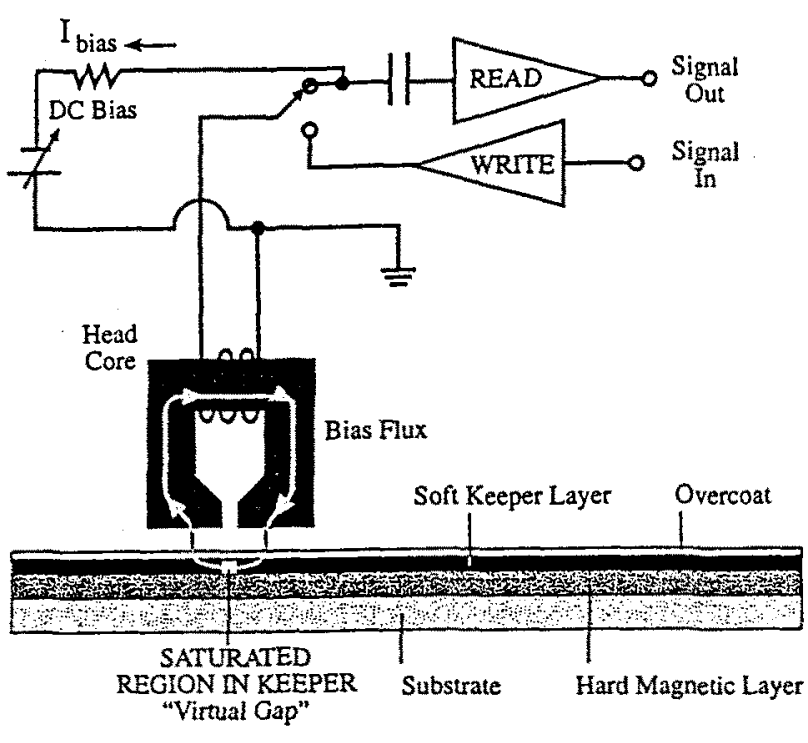

Figure 1. Keepered recording system diagram. Read connection provides dc bias current in the head coil to produce virtual gap in keeper layer

superparamagnetic limit, magnetization decay can occur just from thermal excitation. The keeper dramatically reduces the internal demagnetizing fields and thus reduces the rate of decay. The keeper also reduces the extent of sidewriting effects and is thus very compatible with recording at low bit-aspect ratio and very high track-density. Current activity has thus focused on the compatibility of keepered media with magnetoresistive heads and on the possibility that keepered recording may ultimately allow higher areal densities to be achieved.

\section{PRINCIPLES OF KEEPERED RECORDING}

\section{A. Fundamental Concepts}

The most common configuration for keepered recording consists of a ring head and a medium made up of a magnetically hard recording layer and a soft "keeper" layer. Figure 1 represents schematically a typical system. The recording and keeper layers are separated by a thin break layer to prevent exchange coupling between the two layers. The soft layer should have reasonably high permeability and have a thickness saturation-magnetization product similar to the thickness remanence product of the hard layer.

Operation of keepered recording is best understood in terms of a "virtual gap" induced by the DC bias current applied during readback. The virtual gap forms in the soft layer in a region under the head gap where the applied bias field is strong enough to saturate the soft layer as shown in Figure 1. The incremental permeability in the saturated virtual gap is very low and, from the perspective of the signal flux from the hard layer, it appears as a magnetic gap. This virtual gap is fixed in position relative to the head as it scans over the recorded data. The resulting geometry may thus be thought of as a composite head made up of the ring head plus the soft layer with its virtual gap. This composite head has infinitely long pole lengths and is in very close contact with the recording medium. In theory therefore, the readback spacing loss is negligible. The overall efficiency of the composite head is reduced by the reluctance of the two additional intemal gaps (between the keeper and the pole-tips). If the keeper is highly permeable and the pole-tips are long the reduction in efficiency will be small.

\section{B. Write Process}

In keepered recording, the analysis of the write process is complicated by the saturable, soft magnetic keeper layer. Ideally, a complete, self-consistent write model, incorporating head, keeper, and recording layer behavior should be used. Considerable insight can be developed by using a simpler, Williams-Comstock [16] approach to find the final arctangent transition parameter, $a_{2}$. First $a_{1}$ is calculated, this is the initial " $\mathrm{a}$ " parameter still in the presence of the head writing field. The value $a_{1}$ then relaxes to $a_{2}$ as the transition moves away from the head. In ultra-high density recording applications, $a_{1}$ is limited by the head field gradient at the center of the recording layer at the point where the transition is written, which is where the head field equals the coercivity. It is assumed that the keeper layer has very little effect on this initial write process because it is heavily saturated in the vicinity of the transition. The keeper layer, however, will significantly affect the relaxation from $a_{1}$ to $a_{2}$. Normally the transition's own demagnetizing fields will cause it to broaden as it moves away from the head. In the presence of the keeper layer, the demagnetizing fields are significantly reduced and the relaxation from $a_{1}$ to the final $a_{2}$ is less pronounced.

Modifying the augmented Williams-Comstock derivation (Eqn. (5) in [17]) to include the presence of the keeper, we get the following equation which can be solved iteratively for $\mathrm{a}_{2}$ :

$$
\frac{\mathrm{a} 2}{\mathrm{a} 1}=\frac{1+2 \cdot \pi \cdot \chi \cdot \mathrm{t}\left(\frac{1}{\mathrm{a} 2+\frac{\mathrm{t}}{2}}-\left(\frac{\mu-1}{\mu+1}\right) \cdot\left(\frac{1.5+\frac{\beta}{\mathrm{t}}}{\mathrm{a} 2+1.5 \cdot \mathrm{t}+\beta}+\frac{.5-\frac{\beta}{\mathrm{t}}}{\mathrm{a} 2+.5 \cdot \mathrm{t}-\beta}\right)\right.}{1-\chi \cdot(1-\mathrm{S}) \cdot \frac{\mathrm{Hc}}{\mathrm{Mr}}}
$$

where $\chi \cong M_{r} / 4 \mathrm{H}_{c}$ is the Williams-Comstock approximation for the minor loop susceptibility of the recording layer, $t$ is the hard layer thickness, $\mu$ is relative permeability of the keeper layer, $\beta$ is break layer thickness, and $S^{*}$ is the coercivity squareness of the recording layer. 


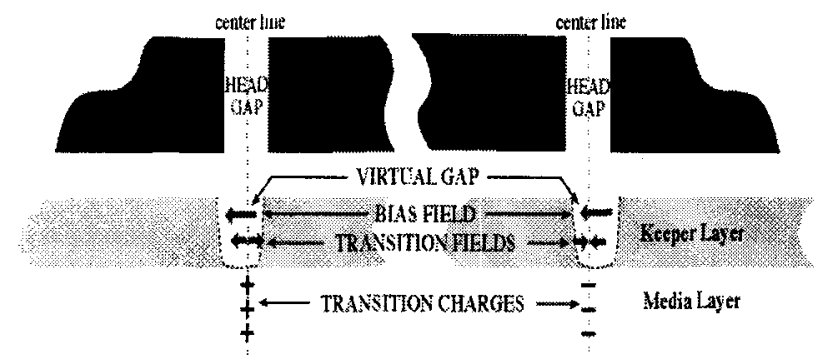

Figure 2. Illustrating the origins of the time and amplitude asymmetries in keepered recording.

It is assumed that the keeper layer is infinitely thick, and the image fields from the keeper are determined by Potter's expression for the field from an arctangent transition [18].

Assume a $15 \mathrm{~nm}$ keeper with permeability of 100 , a break-layer of $1 \mathrm{~nm}$, and a typical $100 \mathrm{Kbit} / \mathrm{inch}$ inductive head recording system: $\mathrm{M}_{\mathrm{r}} \mathrm{t}=1.9 \mathrm{memu} / \mathrm{cm}^{2}$ $\left(\mathrm{M}_{\mathrm{r}}=560 \mathrm{emu} / \mathrm{cc}, \mathrm{t}=34 \mathrm{~nm}\right), \mathrm{H}_{\mathrm{c}}=2000 \mathrm{Oe}, \mathrm{S}^{*}=0.8$. Because of the thickness of the keeper and break layer, the initial $a_{1}$ parameter is $66 \mathrm{~nm}$ for keepered recording vs. $59 \mathrm{~nm}$ for no keeper. However, after the relaxation, from (1), we have $a_{2}=73 \mathrm{~nm}$ for the keepered medium vs. $74 \mathrm{~nm}$ for no keeper. The additional spacing caused by the keeper is thus almost exactly counterbalanced by its effectiveness in reducing the subsequent broadening of the transition.

\section{Read Process}

The two basic approaches for analyzing the read process, reciprocity and direct calculation of read flux in the head, are also applicable to keepered recording.

Analysis by reciprocity requires linearity. Because the keepered recording system is inherently non-linear, the read process must be linearized by a small-signal expansion about the bias current, $\mathrm{I}_{\mathrm{bias}}$. Using a simple finite difference model, Gooch, et al., [2] calculated $\partial \mathrm{H}_{\mathrm{x}}(\mathrm{x}) / \partial \mathrm{I}_{\text {bias }}$ around the optimum bias point and showed significant sharpening of the small-signal head sensitivity function.

Study of the large-signal read process involves direct calculation of the flux in the head while including nonlinear saturation effects in the keeper layer. Loven, et al., [9] used a non-linear boundary element method to study readback pulses from isolated step transitions of magnetization in a longitudinal recording medium. They found that the pulse sharpening of keepered recording observed by Gooch, et al., can be accompanied by time-shifts and time asymmetries. Work by Coughlin et al. [19,20] also revealed asymmetric pulse amplitude.

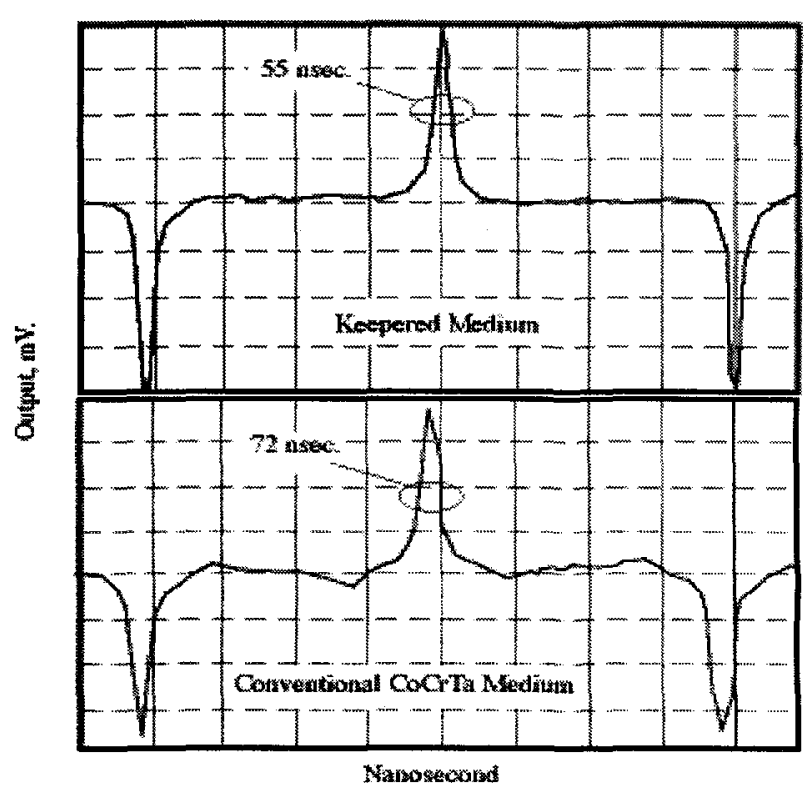

Figure 3. Isolated pulse responses for keepered vs. unkeepered media (copyright 1993 IEEE [7])

These nonlinearities are apparently caused by the relatively large fields produced in the keeper layer by the recorded transition, Figure 2. The longitudinal component of these fields changes sign at the transition location. Consequently, it adds to or subtracts from the bias field to change the shape and location of the virtual gap. The result is a disturbance of the readback pulse timing and amplitude depending on the sign and location of the recorded transitions. Mian, et al. measured the timing shifts in a keepered recording system using timedomain correlation techniques and found the shifts to be a significant fraction of a bit-cell [8]. More recently, Wilson, et al. have modeled these effects as arising from a signal-dependent modulation of the head efficiency. Based on this model, they have been able to derive an effective nonlinear equalization technique [11].

\section{STRUCTURE AND FABRICATION OF KEEPERED DISKS}

Keepered disks are typically composed of a conventional longitudinally-oriented hard medium overlain by a magnetically soft (high permeability) layer. The soft film is deposited by RF or DC magnetron sputtering. Early longitudinal keepered media work was done using permalloy (NiFe alloy) films [2][21][8]. More recently, very thin DC or RF magnetron sputtered films of Sendust (Fe-Si-Al alloys) have been found to give better performance $[22][19][20][23]$. Other soft magnetic materials have been explored as keeper layers including $\mathrm{Co}-\mathrm{Zr}-\mathrm{Nb}$ alloys[10]. It has been found to be beneficial to have a non-magnetic layer between the hard and soft films. 


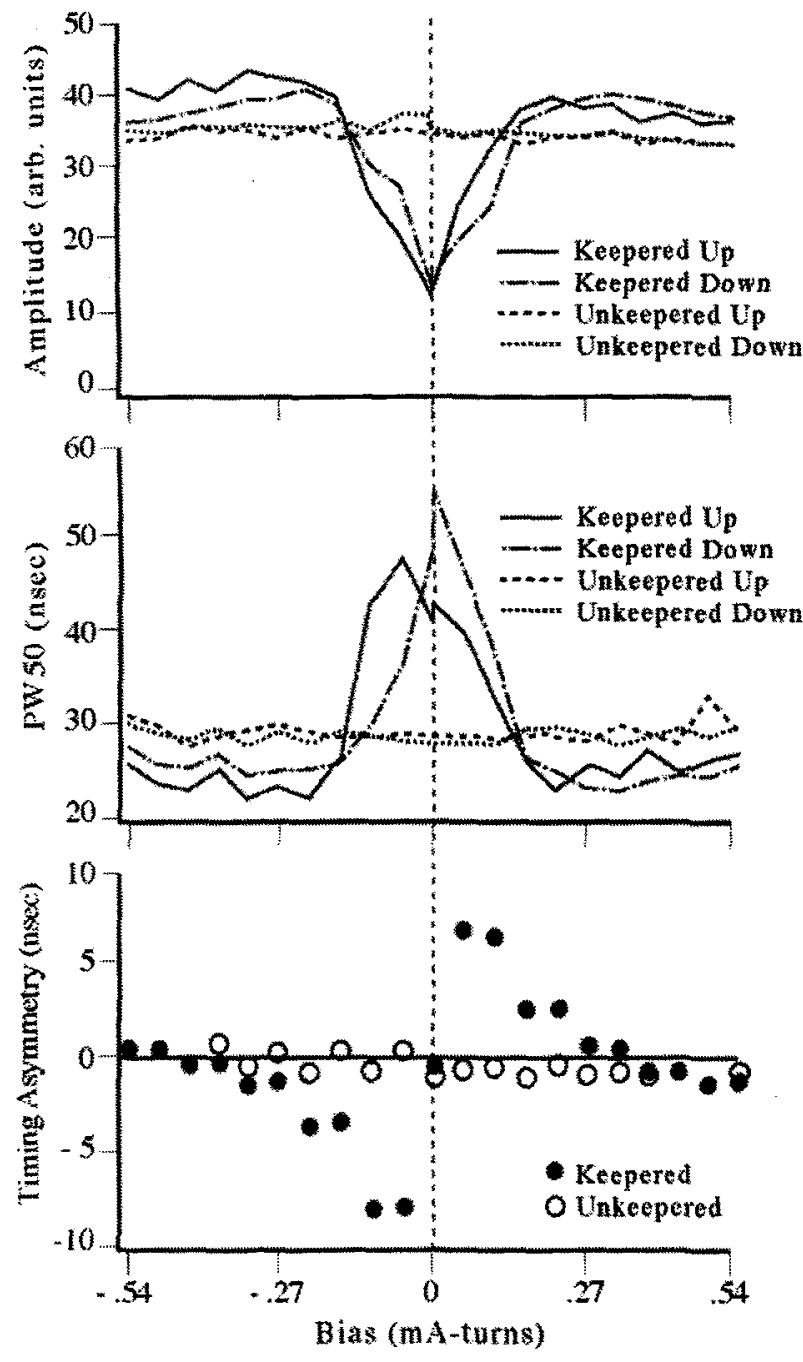

Figure 4. Amplitude, PW50, and timing as a function of bias MMF. (after [19])

This "exchange break" ensures that the soft film has low coercivity (high permeability) and responds only to magnetostatic coupling. Typical materials include DC magnetron sputtered $\mathrm{Cr}, \mathrm{C}$ or $\mathrm{Si}$ or $\mathrm{RF}$ sputtered insulators such as $\mathrm{Al}_{2} \mathrm{O}_{3}$. A layer as thin as 1.5 to $3 \mathrm{~nm}$ is found to be effective.

The best inductive head performance is observed when the saturation-thickness product, $M_{s} t_{k}$, for the keeper is comparable to or slightly less than the remanencethickness product of the hard layer, $M_{\mathrm{r}} \mathrm{t}_{\mathrm{h}}$. Sendust films which have been circumferentially oriented are found to give more output than radially oriented films Measured coercivities, which are around $40 \mathrm{Oe}$ on a magnetooptical magnetometer, are several times higher than for similar films deposited without the hard layer.

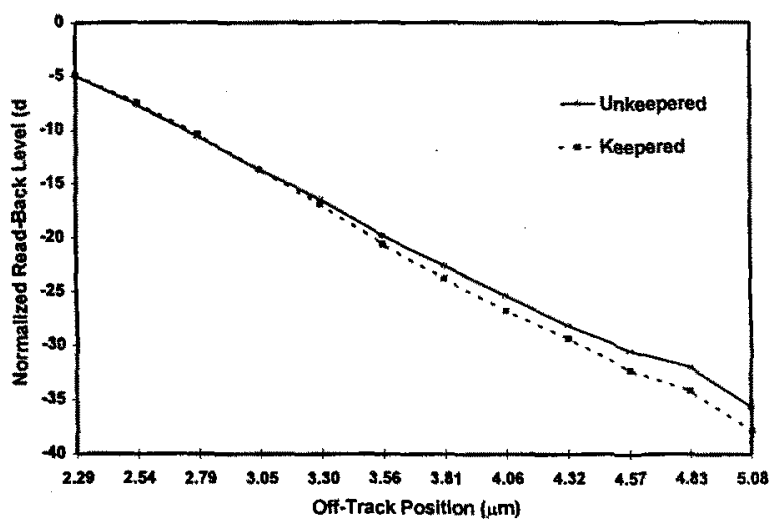

Figure 5. Readback signal vs. displacement off track for keepered and unkeepered media. The density is 400 flux-changes $/ \mathrm{mm}$. The head has a track width of about 3 microns.

\section{INDUCTIVE HEAD MEASUREMENTS AND RESULTS}

\section{A. Linear Density Effects}

Figure 3 compares isolated pulses for keepered vs. unkeepered media used with an inductive head (from $\mathrm{Kao}$ et al. [7]). This early work illustrates several features which quickly attracted attention: larger signals, better resolution, and the elimination of the troublesome "undershoots" for the outside edges of the poles-tips.

The gains from keepered recording are a strong function of the bias field. Figure 4 shows the gain in amplitude and resolution as a function of bias. This figure also shows that the gains are accompanied by significant asymmetries in amplitude and timing. At zero bias there is only a very small diffuse response. As the bias is increased, the readback pulse starts to rise but exhibits large timing asymmetry. As the bias is increased further, the PW50 reaches a minimum and the amplitude reaches a maximum. Just beyond this point, the asymmetry becomes smallest and the best system errorrates are obtained.

\section{B. Off-Track Effects}

During writing, the fields near the head-gap are largely unaffected by the presence of the keeper, which saturates heavily. Off the sides of the head, however, the reluctance of the intervening air gap and the shunting effect of the keeper cause the fields to drop more rapidly than normal. Measurements of trackprofiles reveal a reduction in write-width of 5\% [20]. More significantly, the side erase-bands [24] have been measured to drop by a factor of two (see section VII B). 


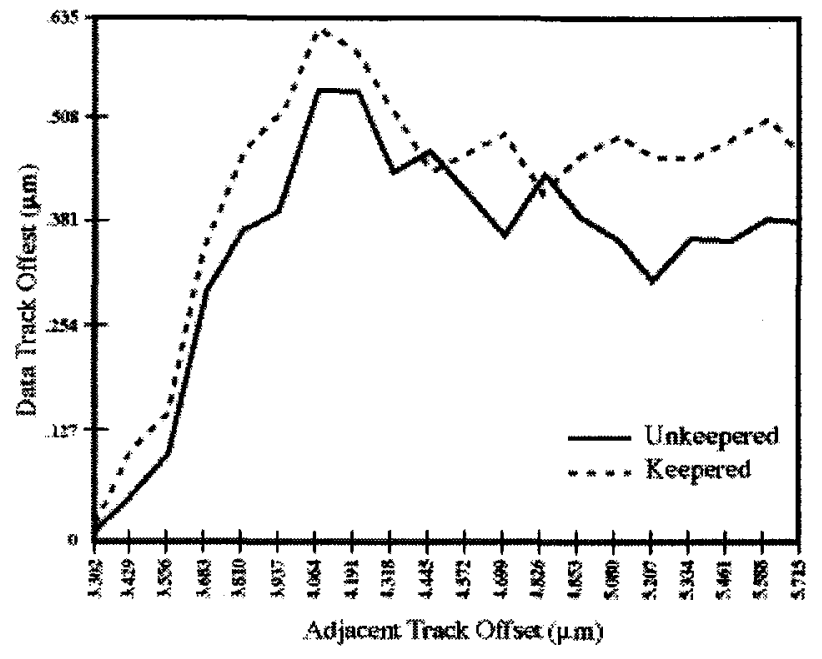

Figure 6. "747" curve showing off-track capability vs. adjacent-track squeeze for a PRML channel. The keepered disk is tested at $137 \mathrm{kbpi} \mathrm{versus} 112 \mathrm{Kbpi}$ for the unkeepered (after [20])

On readback, it has been noted that the virtual gap for an inductive head has a finite width (cross-track) as well as length (down-track). Off the ends of the virtual gap the keeper is unsaturated and highly permeable and might be expected to significantly reduce sidereading in a similar manner to that proposed by Mallory [14] although with an over-layer keeper. Figure 5 is a plot of sidereading attenuation vs. displacement off track for keepered and unkeepered media. The head has a track width of about 3 microns. In the region of interest, 20$30 \mathrm{~dB}$ of attenuation, however, the level of sidereading is only reduced by about $\sim 1.5 \mathrm{~dB}$.

\section{Measured Areal Density Improvement}

Error-rate measurements with peak detect channels have shown linear density improvements of $20 \%$ for keepered media [20]. For a PR4ML channel, Figure 6 shows a socalled " 747 " off-track margin plot [24] for keepered and unkeepered media at a $10^{-5}$ bit error rate [20]. Although the keepered media is recorded at $23 \%$ higher linear density, its off-track margin and squeeze failure point are the same or better than for unkeepered media. The head is a 42-turn planar head with a gap-length of 0.2 $\mu \mathrm{m}$, a track-width of $3.5 \mu \mathrm{m}$, and a $27 \mathrm{~nm}$ flying height. The disk is CoCrTa with a coercivity of 2200 Oe and a $\mathrm{M}_{\mathrm{r}} \mathrm{t}$ of $2.25 \mathrm{memu} / \mathrm{cm}^{2}$. The added keeper layer is $15 \mathrm{~nm}$ of sputtered Sendust.

Figure 7 shows "bath-tub" error rate plots comparing keepered and unkeepered media for a 42-turn, thin-film proximity recording head taken on a spin-stand and detected with an EPR4 channel emulation. The head and disk parameters are the same as described above, except that the flying height is $25 \mathrm{~nm}$ and the trackwidth is $3.2 \mu \mathrm{m}$. The results are taken at $12 \mathrm{~m} / \mathrm{s}$. Gap-

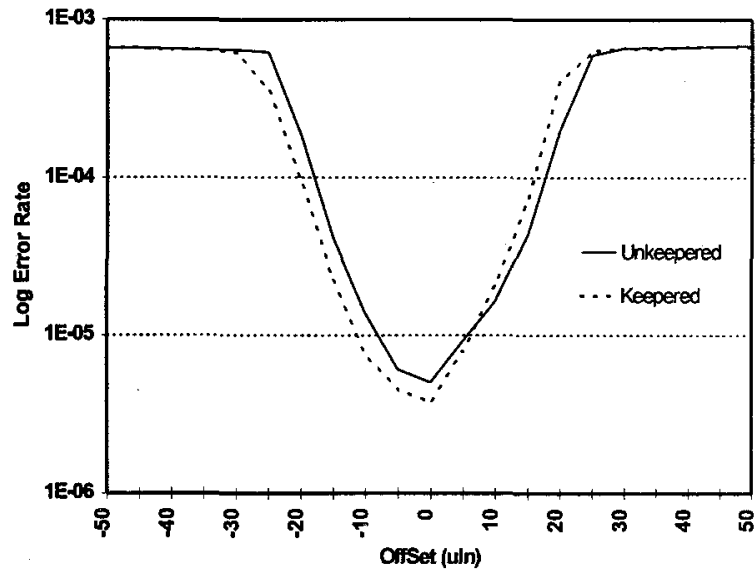

Figure 7. Error-rate vs. off-track position with an EPR4 $(0,4,4)$ channel for keepered and unkeepered disks. The keepered disk is operating at $24 \%$ higher linear density than the unkeepered disk.

length is $0.2 \mu \mathrm{m}$. The results are taken with no adjacent interfering tracks - just a dc-erase background. It is to be noted that the keepered disk was evaluated at $24 \%$ higher linear density (constant velocity) and yet had the same off-track performance capability.

\section{E. Nonlinear Equalization}

As mentioned above, keepered recording exhibits distinctive nonlinear effects. If these could be compensated, the readback process could operate at lower bias currents where the gains in amplitude and resolution are greatest. Wilson et al.[11] have proposed using a non-linear equalization technique to correct keepered media timing and amplitude asymmetries. The readback waveform is first integrated, passed through a memoryless nonlinearity, then differentiated to recover a corrected version of the original waveform. Dipulse extraction before and after equalization show that this non-linear equalization technique eliminates almost all the nonlinear distortion. Figure 8 reproduced from [11] shows the Sampled Amplitude Margin plot [25] for a PRML channel before and after this linearization. An improvement of approximately $10 \%$ in margin is observed. Further results from Hoinville [31] suggest that the onset of partial erasure may be moved to higher density by the presence of the keeper.

\section{OTHER KEEPERED CONFIGURATIONS}

Almost all the work done to date on keepered recording has employed an inductive ring head and has placed the keeper layer on top of the hard layer. There are, however, a number of other possible configurations which are also of interest: keeper layer under instead of over the hard layer, MR readback instead of inductive, perpendicular hard layer instead of longitudinal. 


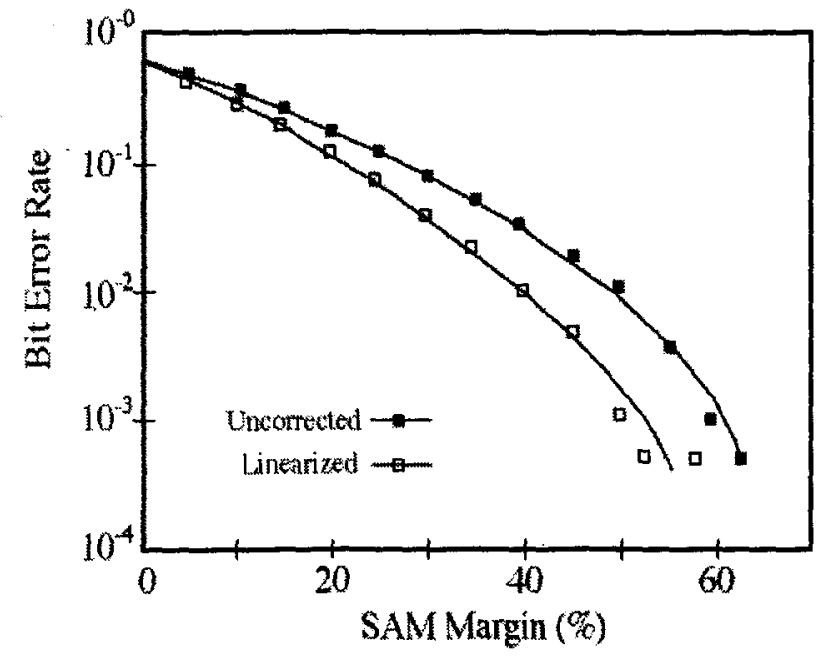

Figure 8. Sampled Amplitude Margin plot before and after nonlinearity correction (after [11])

It was acknowledged in the original work that the keeper may perform better underneath rather than on top of the hard layer [1]. For practical reasons of fabrication, however, all of the early work was done with the keeper layer on top of the hard layer. Only recently, in conjunction with MR heads (Section VII) has it been demonstrated that the "keeper under" configuration is also very viable.

With respect to magneto-resistive heads, one might suspect by analogy with the inductive ring head that a single-gap yoke type MR sensor should work well with keepered media. However, it is not as clear that a conventional double-gap shielded MR head will work well. Again the recent results described in the following section show that good signals can be obtained from a conventional double-shielded MR head without any further special provisions for bias fields.

Perpendicular recording with a keeper also raises a host of possible configurations: single-layer, dual-layer, polehead, ring-head, MR-head, keeper-over, keeper-under. However, none of these configurations seem particularly promising. Underlying all the options is the concern that the keeper will saturate heavily in the vicinity of a relatively isolated transition. In longitudinal recording the thickness of the keeper layer is chosen to just avoid the keeper becoming saturated, i.e. $\mathrm{M}_{\mathrm{s}} \mathrm{t}_{\mathrm{soft}}>\mathrm{M}_{\mathrm{r}} \mathrm{t}_{\text {hard }}$. For perpendicular recording, however, the flux flowing back across an isolated transition through an ideal nonsaturating soft film would tend to infinity. It is thus likely that the readback process would be highly nonlinear. No work on keepered perpendicular media has yet been reported.

\section{MR HEAD MEASUREMENTS AND UNDERLAYER KEEPER RESULTS}

The theory of a virtual gap can also be applied to readback with a conventional double-shielded softadjacent-layer magneto-resistive element. Acting as a one-turn head, the normal levels of MR bias current should create sufficient field across the gap between the shields to saturate the keeper layer. In this case however, the unsaturated areas of keeper either side of this virtual gap are now effectively extensions of the shields. The equivalent magnetic structure is now an MR head with its shields in contact with the hard recording layer but with a deeply recessed sensor. Furthermore, in contrast to an inductive head, the virtual gap will extend the full width (across-track) of the shields and will thus be much greater than the width of the MR element. With this model in mind, we expect MR heads to show similar improvements in resolution but to have reduced output levels and to show no improvement in sidereading.

Tests with MR heads confirm that signals can easily be recovered from conventional "overlayer" keepered media at normal bias currents. The observed improvements in resolution are not large but neither are the reductions in amplitude. Interestingly, as for inductive heads, we have observed similar reductions in effective written track width on the keepered media.

As indicated above, the keeper layer can be either above or below the hard magnetic layer. With MR heads, better results have been obtained with the keeper below the hard layer. Figure 9 shows that significant reductions in PW50 can be obtained with no loss in amplitude. These results were obtained with and without an underlayer keeper with an $\mathrm{M}_{\mathrm{s}} \mathrm{t}_{\mathrm{k}}$ of 0.7 $\mathrm{memu} / \mathrm{cm}^{2}$. The hard layer was CoCrTa with $\mathrm{H}_{\mathrm{c}}=18000 \mathrm{e}$ and $\mathrm{M}_{\mathrm{r}} \mathrm{t}=0.7 \mathrm{memu} / \mathrm{cm}^{2}$. An MR head with a wide shield-shield gap of $1.1 \mu \mathrm{m}$ was used. An $18 \%$ reduction in PW50 can be seen with the underlayer keeper. The reduction of the skirts of the isolated pulses is even larger. Improved noise performance is also observed with the reduction principally at lower frequencies [26].

\section{SCALING AND RECORDING LIMITS}

There is a fundamental limit to recording density known as the superparamagnetic limit. At this limit, the thermal activation energy is comparable with the energy required to switch the magnetic grains of the recording medium. In this situation, the recorded information will decay rapidly with time [27]. This decay is more severe if there are large internal demagnetizing fields within the 


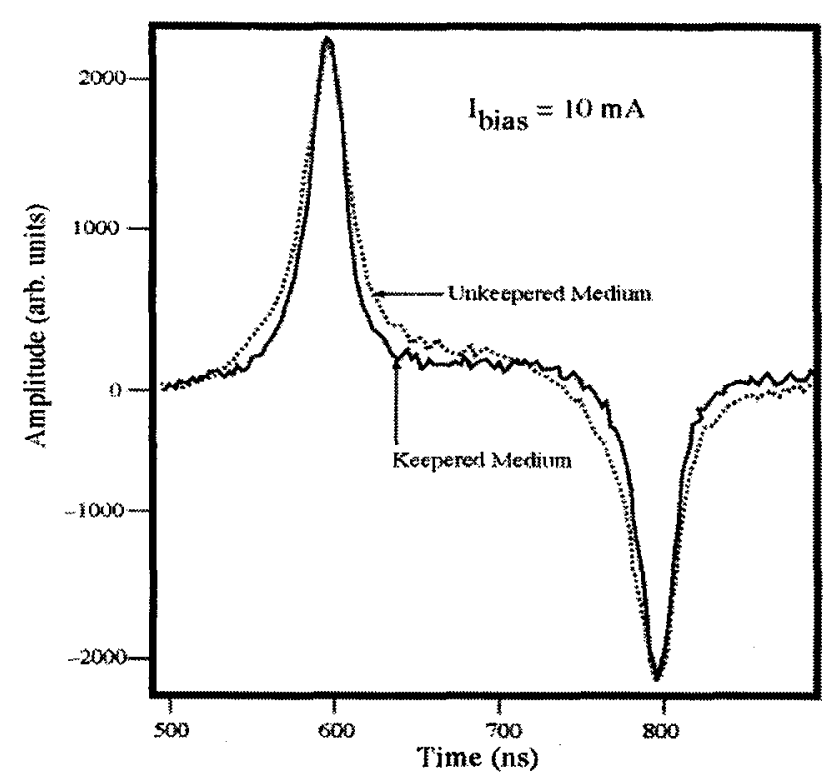

Figure 9. Isolated pulses for MR readback of keepered media where the keeper layer is placed below the hard recording layer.

medium. The limit does permit a trade-off between signal-to-noise and ultimate density. Because of this, it is found more advantageous to operate on very narrow tracks at low bit aspect ratios [30].

\section{A. Thermal Stability}

To obtain large outputs from traditional recording systems every effort is made to maximize the field strength coming out of the surface of the medium.

Unfortunately, high external fields imply high internal demagnetizing fields. In both vertical and longitudinal systems the internal fields approach the coercivity of the medium. It is quite easy therefore for the recorded patterns to demagnetize further under the influence of stray fields, mechanical stress, or, in the ultimate limit, just due to thermal activation [27].

In contrast, the storage mode for keepered recording is intrinsically very stable. Large demagnetizing fields are only present during writing and reading. At all other times the keeper layer acts to dramatically reduce the demagnetizing fields.

Figure 10 shows thermal decay rates for keepered and unkeepered media measured by J. Chen et al. from the University of Minnesota [10]. The results were taken at 5000 flux-changes $/ \mathrm{mm}$ on media with a low $\mathrm{M}_{\mathrm{r}} \mathrm{t}$ of 0.6 $\mathrm{memu} / \mathrm{cm}^{2}$. The unkeepered media shows a decay in signal of $2.8 \%$ per decade of time vs. $1.1 \%$ per decade of time for the keepered media.

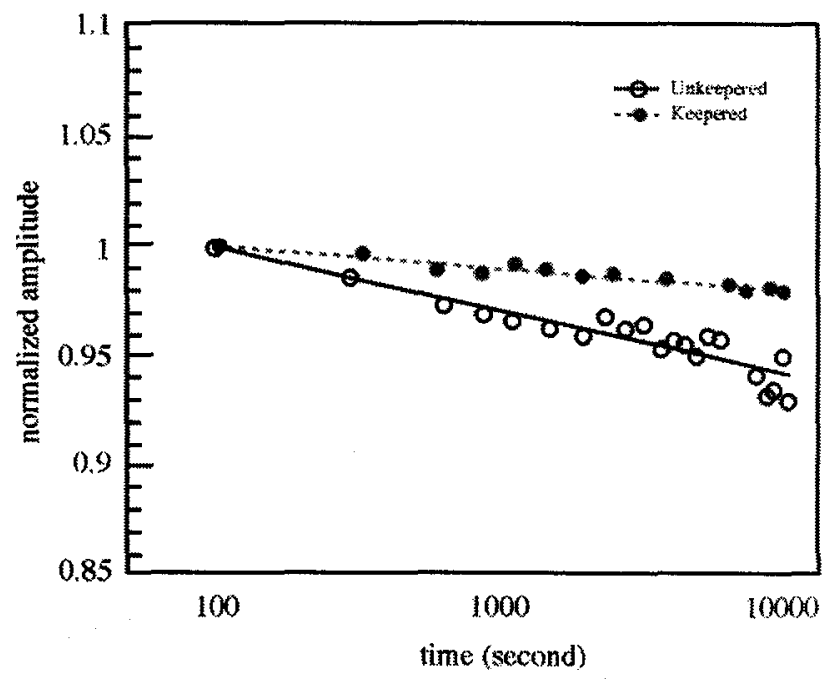

Figure 10. Measured thermal decay for keepered and unkeepered $0.6 \mathrm{memu} / \mathrm{cm} 2 \mathrm{Mrt}$ media at $5000 \mathrm{frpmm}$ (150 kfci). (copyright 1997 IEEE [10])

\section{B. Bit aspect ratio}

The so-called "side-erase-band" [24] is an important attribute of longitudinal magnetic recording. The absence of this erase-band has been cited as one of the reasons that perpendicular recording has not been adopted commercially [28]. However, for conventional longitudinal recording at very low bit aspect ratio, the erase-band may become proportionately too wide since it tends to scale with linear-density rather than track density. Keepered recording offers a significant reduction in the width of the side erase band and is thus very compatible with recording at low bit-aspect ratio and very high track-density.

Measurements, using $120 \mathrm{~mA}$ pk-pk into a 15 turn head with a 0.7 micron gap showed a reduction in side-erase bands from 0.46 micron to 0.24 micron in going from unkeepered to keepered media. This result was measured at 1700 flux-changes $/ \mathrm{mm}$ using the tripletrack profile technique [32]. The medium had a coercivity of 1450 Oe and an Mrt of $0.8 \mathrm{memu} / \mathrm{cm}^{2}$.

\section{CONCLUSIONS AND OUTLOOK}

Keepered recording is a novel recording technique about which there is undoubtedly still much to learn. The technique takes advantage of the nonlinear properties of a soft film which is incorporated into the medium. This approach opens up possibilities for increasing the resolution of the readback process in both the alongtrack and across-track dimensions. With inductive heads, significant improvements in resolution and performance have been demonstrated as well as small improvements in the cross-track direction. With 
magneto-resistive heads the best results are being obtained with an underlayer keeper. There are a variety of other possible configurations and ways in which keepered recording might play a role. As well as offering superresolution and reducing the effects of readback spacing, perhaps the most exciting aspect of keepered recording lies in its potential at very high density. The lower rates of thermal decay and the smaller side erase-bands suggest that keepered media may be able to play an important role in extending magnetic recording to the highest areal densities.

\section{REFERENCES}

[1] R. Wood et al, "Magnetic Recording Medium having Magnetic Storage and Saturable Layers, and Apparatus and Method using the Medium", US Patent 5,041,922, August 20th 1991.

[2] B. Gooch et al., "A High Resolution Flying Magnetic Disk Recording System with Zero Reproduce Loss", IEEE Trans. Magn. Vol. 26, No. 7, pp. 4549-54, Nov. 1991.

[3] B. Gooch, "Magnetic Saturation Controlled Scanning Magnetic Transducer", US Patent 5,119,255, June 2nd, 1992.

[4] B. Gooch, "Solid-State Scanning using Transducer that Utilizes Low Flux Densities", US Patent 5,130,876, July 14th, 1992.

[5] B. Gooch, "Magnetic Control of a Transducer Signal Transfer Zone to effect Tracking of a Path along a Record Medium", US Patent 5,189572, February 23rd 1993.

[6] B. Gooch, "Method and Apparatus for Transferring Information between Two Bodies using a Third Body of Magnetic Material", US Patent 5,153796, October 6th, 1993.

[7] A. Kao et al., "A High Performance laminated, CoCrTa Media with Soft Adjacent Layers for Magnetic Recording", IEEE Intermag Conference, 1993.

[8] G. Mian et al., "Effects of Highly Permeable Keeper Layer on Transition-Shifts for Thin-Film Media", IEEE Trans. Magn. Vol. 30, No. 6, pp. 3984-86, Nov. 1994.

[9] J. Loven et al., "Modeling Study of Isolated Readback Pulses from Keepered Longitudinal Thin-Film Media Using Boundary Element Method", IEEE Trans. Magn. Vol. 30, No. 6, pp. 4275-77, Nov. 1994.

[10] J. Chen, et al., "Experimental Study of Recording Performance of Sendust and CoZrNb Keepered Thin Film Media," IEEE Intermag Conference, 1997.

[11] B. Wilson et al., "Read-Back Nonlinearity in Longitudinal Keepered Recording”, IEEE TMRC ‘97, September 1997.

[12] N. Sawazaki, "Magnetic Recording and Reproducing Apparatus", US Patent 4,530,016, July 16th 1985.

[13] N. Sawazaki, "Magnetic Recording and Reproducing Apparatus", US Patent 4,535,369, August 13th, 1985.

[14] M. Mallary, "Magnetic Medium for Longitudinal Recording", US Patent 5,176,965, January 5, 1993.
[15] Y. Murakami et al., "Magnetic Super-resolution", IEEE Trans. Magn., Vol. 31, No. 6, pp. 3215-3217, Nov. 1995.

[16] M. Williams and L. Comstock, "An Analytical Model of the Write Process in Digital Magnetic Recording," 17th Annu. AIP Conf. Proc., Part. 1, No. 5, pp. 738-42, 1971.

[17] J. Goldberg, et al., "Calculating Final Transition Widths using an Augmented Williams-Comstock Model" IEEE Trans. Magn., Vol. MAG-32, No.-5, pp. 3998-4000, Sept. 1996.

[18] R. I Potter, "Analysis of Saturation Magnetic Recording based on Artangent Magnetization Transitions", J. Appl. Physics, Vol 41, pp. 1647-51, March 1970.

[19] T. Coughlin et al., "Magnetic Recording Performance of Keepered Media", J. Appl. Phys. Vol. 81, No. 8, pp. 4693-4695, 15 April 1997.

[20] T. Coughlin et al., "High Density Recording with Keepered Media and Planar Heads" J. Appl. Phys. Vol. 81, No. 8, pp. 48434845, 15 April 1997.

[21] K. Sin et al., "Comparison of Reproduce Signal and Noise of Conventional and Keepered $\mathrm{CoCrTa} / \mathrm{Cr}$ Thin-Film Media", J. Appl. Phys., Vol. 75, No. 10, pp. 6150-52, May 1994.

[22] W. Reed et .al., 'Four Discrete Magnetic States of Keepered Media," J. Appl. Phys., Vol. 81, No. 8, pp. 4683-85, April 1997.

[23] B. Lal et al., "The Effects of Sendust Keeper, Cr Break-Layer, Thicknesses and Reproduce Bias on Keepered Media Peformance and Pulse Asymmetry", IEEE InterMag Conference New Orleans, 1997.

[24] Kanu Ashar, Magnetic Disk Drive Technology, IEEE Press, New York, 1997, pp. 257-259.

[25] A. Taratorin, "Margin Evaluation of PRML Channel: Nonlinear Distortions, Misequalization, and Off-Track Noise Performance", IEEE Trans. Magn., Vol. 31, No. 6, pp. 3060-3065, Nov. 1995.

[26] M. Nichols et al., "Under-Keepered Media with MR heads" submitted to IEEE Intermag Conference, San Francisco, 1998.

[27] Pu-Ling Lu and Stanley H. Charap, "High Density Magnetic Recording Media Design and Identification: Susceptibility to Thermal Decay," IEEE Trans. Magn., Vol. 31, No. 6, pp. 2767-69, Nov. 1995.

[28] T. Beaulieu et al., "Track Density Limitations for Dual-Layer Perpendicular Recording in a Rigid Disk Environment", IEEE Trans. Magn. Vol. 25, No. 5, pp. 3369-3371, Sept. 1989.

[29] T. Coughlin et al., "Write Effects with Keepered Media", submitted to IEEE InterMag 1998.

[30] H. N. Bertram et al., "Signal to noise and density limit estimates in longitudinal magnetic recording", submitted to IEEE InterMag 1998.

[31] J. Hoinville \& W. Haines, "Noise Characteristics of Keepered Magnetic Recording Media", IEEE Intermag Conference, New Orleans, 1997.

[32] T. Lin et al., "Effects of Frequency, Current, and Head Type on Read, Write, and Erase-width of Narrow-Track Heads", IEEE Trans. Magn., Vol. MAG-25, No. 1, pp 710-715, Jan. 1989. 